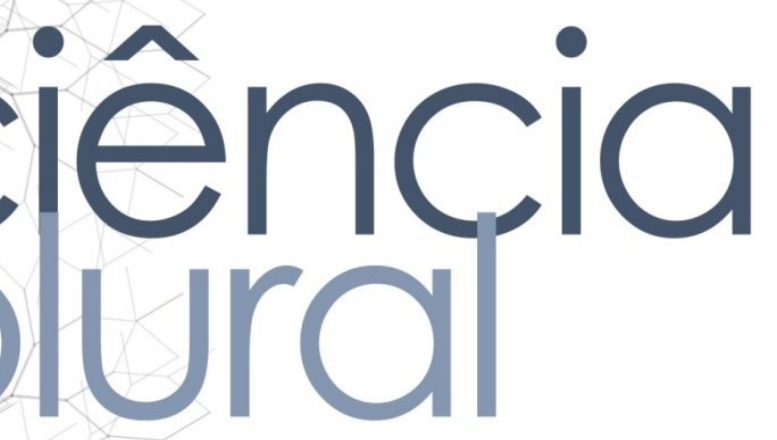

\title{
ASSISTÊNCIA À SAÚDE DA CRIANÇA: UMA ANÁLISE MULTIDIMENSIONAL DOS SERVIÇOS DE SAÚDE
}

\section{Child health assistance: a multidimensional analysis of health services}

Marcelo de Araújo Abreu Pereira • Acadêmico do Curso de Graduação em Enfermagem da Faculdade de Ciências da Saúde do Trairi (FACISA) / Universidade Federal do Rio Grande do Norte (UFRN). E-mail: marcelo_araujo150@hotmail.com

Dandara Rayssa Silva de Souza - Enfermeira pela FACISA/UFRN. E-mail: dandara_rayssa@hotmail.com

Ana Mayara Gomes de Souza • Enfermeira. Mestranda do Programa em Pós-Graduação em Saúde Coletiva na UFRN. E-mail: ana.mayara@yahoo.com.br

Tainara Lorena dos Santos Ferreira - Enfermeira. Mestranda do Programa em Pós-Graduação em Saúde Coletiva na UFRN. E-mail: tainara_lorena@hotmail.com

Fábia Barbosa de Andrade • Enfermeira. Doutora em Ciências da Saúde. Professora Adjunto IV da UFRN. E-mail: fabiabarbosabr@gmail.com

Autor responsável pela correspondência:

Marcelo de Araújo Pereira. Email: marcelo_araujo150@hotmail.com. 


\section{ciência \\ plural}

\section{RESUMO}

Introdução: A saúde infantil constitui-se como ações e práticas com intuito de proporcionar um atendimento integralizado a saúde da criança, vista como area prioritária no âmbito do cuidado. Objetivo: avaliar a qualidade da assistência prestada à criança desde os primeiros momentos de vida até a inserção e acompanhamento no programa de puericultura. Metodologia: Trata-se de um estudo descritivo, avaliativo de abordagem quantitativa, com 200 mulheres mães/responsáveis por crianças menores de dois anos, atendidas em Unidades Básicas de Saúde, no município de Santa Cruz/RN. Resultados: Com relação os cuidados ofertados, observou-se que o apgar entre 7 a 10 no primeiro e quinto minuto foi atribuído na maioria da amostra; Tratandose do estímulo ao vínculo materno/infantil, a maioria das mães afirmou que permaneceu junto ao seu filho no mesmo quarto após o parto; bem como amamentou seu filho logo após o parto. Por fim, sobre as condições de saúde no primeiro mês de vida, a maioria da relatou que não houve internamento por nenhum tipo de complicação. Conclusão: Observou-se que as práticas assistenciais foram realizadas na sua maioria, destacando o comprometimento dos profissionais atuantes nos serviços de saúde, o que confere aos serviços uma boa qualidade frente às demandas solicitadas. Porém as fragilidades e desafios ainda existentes demonstram a necessidade de estratégias para implementação de medidas estratégicas em busca de melhores resultados.

Palavras-chave: Assistência à Saúde. Avaliação em Saúde. Serviços de Saúde Materno-Infantil.

\section{ABSTRACT}

Introduction: Children's health constitute actions and practices aimed at providing an integrated service to the health of the child, as a priority area in the area of care. Objective: to evaluate the quality of care provided to the child from the first moments of life to insertion and follow-up in the childcare program. Methodology: This is a descriptive, evaluative study with a quantitative approach, with 200 mothers / caregivers of children under two years of age attended at the Basic Health Units in the city of Santa Cruz / RN. Results: Regarding the care offered, it was observed that the apgar between 7 and 10 in the first and fifth minutes was attributed in the majority of the sample; As for the stimulation of the maternal / child bond, most mothers said that they stayed with their child in the same room after childbirth; as well as breastfed her child soon after delivery. Finally, about health conditions in the first month of life, most of them reported that there was no hospitalization for any type of complication. Conclusion: It was observed that the assistance practices were mostly carried out, highlighting the commitment of the professionals working in the health services, which gives the services a good quality against the requested demands, but the fragilities and challenges still exist demonstrate the need for strategies to implement strategic measures for better results.

Keywords: Delivery of Health Care. Health Evaluation. Maternal-Child Health Services. 


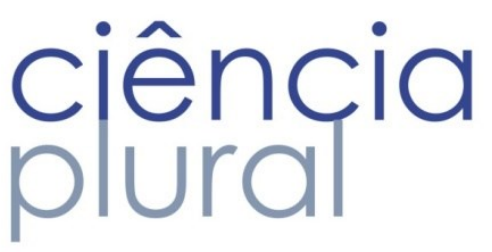

\section{INTRODUÇÃO}

No âmbito do cuidado infantil, prestar uma assistência adequada através dos programas disponíveis no Sistema Único de Saúde apresenta-se como fundamental no contexto da saúde pública, compreendendo uma área de interesse para os órgãos nacionais e mundiais. Brasil ${ }^{1}$ destaca que a absoluta dependência dos adultos, e o fundamental interesse em garantir o desenvolvimento adequado de gerações futuras, com indivíduos mais saudáveis e socialmente adaptados, justificam a atenção as crianças como prioridade nas políticas.

Ressalta-se ainda que a atenção integral à criança significa prover todos os serviços necessários, em busca da resolutividade às demandas de sua saúde, seja a partir da Atenção Primária à Saúde (APS), atenção especializada ambulatorial e hospitalar, na atenção à urgência e emergência, nos serviços especializados e internação hospitalar ${ }^{1}$. No Brasil, observa-se, grande variação no cuidado rotineiro prestado no nível hospitalar logo após o nascimento, sendo esta variação discutida em várias situações, e com diferentes impactos sobre a saúde do recém-nascido².

Atualmente tem-se buscado a diminuição de intervenções desnecessárias, em busca de fortalecer a interação do binômio mãe e filho. Relacionado ainda a práticas imediatas após o nascimento, como amamentação na primeira hora após o nascimento. Estudo ${ }^{3}$ destaca que o início precoce da amamentação foi associado a um risco reduzido de mortalidade, mesmo quando a análise foi restrita a bebês com baixo peso ao nascer, bem como destaca-se o contato precoce pele-a-pele como um momento natural, belo e exclusivo, de reconhecimento familiar, permeado de significados e benefícios para os dois seres ali envolvidos: o ser-mãe e o ser-filho 4 .

No âmbito da APS constitui-se como ações e práticas o atendimento integralizado a partir da dimensão biopsicossocial, a promoção da educação em saúde, a realização do exame físico e da antropometria, a leitura de exames da triagem neonatal, a verificação e o aprazamento do cartão vacinal, a promoção de um ambiente privado e seguro para mãe e usuário, e por fim, a criação do vínculo; Tais ações e práticas também emergem como primordiais para um atendimento profissional qualificado 5 .

Historicamente, se observam grandes avanços nos indicadores de saúde da criança que reflete melhoria da promoção à saúde e a qualidade de vida da criança, através do cumprimento de recomendações e implementação de políticas de saúde que gera melhores resultados. Entretanto, Machado et al. ${ }^{6}$, indica que muito se pode realizar para aprimorá-los, a partir da compreensão da visão de quem está sendo assistido, identificando fragilidades e potencialidades a fim de para elaboração de medidas estratégias e efetivas para as práticas de promoção, proteção e recuperação da saúde. 


\section{ciência \\ plural}

Assim, tendo o cuidado como premissa inerente à assistência à saúde infantil, o presente estudo teve como objetivo avaliar a qualidade da assistência prestada a criança desde os primeiros momentos de vida até a inserção e acompanhamento no programa de puericultura.

\section{METODOLOGIA}

Trata-se de um estudo descritivo, avaliativo, de abordagem quantitativa, realizado na cidade de Santa Cruz, Rio Grande do Norte, Brasil, com 200 mulheres-mães/responsáveis por crianças menores de dois anos, atendidas em Unidades Básicas de Saúde (UBS), sendo recorte da pesquisa intitulada Morbidade e mortalidade materna e infantil: um estudo de avaliação na Atenção Primária à Saúde.

A escolha da amostra se deu por amostragem sistemática com base no cadastro da rede de APS. A população foi constituída por mulheres mães de crianças menores de dois anos (crianças com 1 ano, 11 meses e 29 dias); sendo a escolha da amostra a partir da prevalência da mortalidade infantil no Estado do Rio Grande do Norte, adotando um erro permitido de 5\% e grau de confiança de $95 \%$ e um poder de $80 \%$, resultando em 200 mulheres, mães com filhos de menores de dois anos e residentes na cidade de Santa Cruz e cadastradas na Unidade de Saúde da Família -USF.

A pesquisa foi realizada no período de 2012 a 2013, a partir da aplicação do questionário validado pelo Ministério da Saúde e utilizado na pesquisa nacional "Chamada Neonatal", após a aceitação das mulheres em participar, mediante a assinatura do Termo de Consentimento Livre e Esclarecido (TCLE).

Os dados coletados foram armazenados no Statistical Package for Social Sciences (SPSS), versão 22.0, e processados obtendo medidas de tendência central e dispersão. A pesquisa foi realizada após apreciação do Comitê de Ética em Pesquisa da Universidade Federal do Rio Grande do Norte, segundo o que preconiza a Resolução 466/2012 do Conselho Nacional de Saúde sobre a pesquisa com seres humanos, sob número de parecer 311.613.

\section{RESULTADOS}

O presente estudo buscou avaliar a qualidade assistência prestada a criança desde os primeiros momentos de vida até a inserção e acompanhamento no programa de puericultura oferecido na APS, tendo em vista que tais informações são indispensáveis à qualidade do cuidado. Para tanto, considerou-se as necessidades básicas de saúde, de acordo com a demanda e com os níveis de complexidade dos serviços ofertados tendo como base os princípios e diretrizes do Sistema Único de Saúde-SUS. 
Dentre as entrevistadas, a idade das mães variou entre 18 a 53 anos, sendo a faixa etária entre 18 a 30 anos, predominante $(60,7 \%)$ na amostra. Com relação ao grau de escolaridade, $94,0 \%(n=187)$ das mães entrevistadas possuía o ensino fundamental completo e/ou o ensino médio concluído. Relacionado à criança, a idade em meses compreendeu entre 0 a 23, sendo a maioria da amostra entre 0 a 10 meses, como se destaca na Tabela 1.

Tabela 1 - Dados de caracterização da amostra. Santa Cruz, Rio Grande do Norte, Brasil, 2017.

\begin{tabular}{|c|c|c|c|c|c|}
\hline \multirow{2}{*}{ Idade da mãe } & 18 a 30 anos & \multicolumn{2}{|c|}{31 a 53 anos } & \multicolumn{2}{|c|}{ Não responderam } \\
\hline & $60,7 \%(n=119)$ & \multicolumn{2}{|c|}{$39,3 \%(n=77)$} & \multicolumn{2}{|c|}{$8,0 \%(n=4)$} \\
\hline \multirow{2}{*}{ Escolaridade materna } & Não estudou & Fundamental & Médio & Superior & Não Sabem \\
\hline & $1,1 \%(n=3)$ & $57,8 \%(n=115)$ & $36,2 \%(n=72)$ & $4,0 \%(n=8)$ & $5 \%(n=1)$ \\
\hline \multirow{2}{*}{ Idade das crianças em meses } & \multicolumn{3}{|c|}{0 a 10} & \multicolumn{2}{|c|}{11 a 23} \\
\hline & \multicolumn{3}{|c|}{$51 \%(n=102)$} & \multicolumn{2}{|c|}{$49 \%(n=98)$} \\
\hline \multirow{2}{*}{ Sexo da criança } & \multicolumn{3}{|c|}{ Masculino } & \multicolumn{2}{|c|}{ Feminino } \\
\hline & \multicolumn{3}{|c|}{$46,0 \%(n=92)$} & \multicolumn{2}{|c|}{$54,0 \%(n=108)$} \\
\hline
\end{tabular}

Concernente a avaliação da criança na primeira hora de vida, Tabela 2, observou-se sobre o índice de Apgar, que no primeiro minuto foi atribuído a 76,5\%, apgar entre 7 ou 10; e 8 a 10 no $5^{\circ}$ minuto de vida, para $78,0 \%$ das crianças. Com relação às crianças que nasceram com baixo peso, 0 valor correspondeu a $5,5 \%(n=11)$ da amostra total.

Tabela 2 - Avaliação realizada na primeira hora de vida. Santa Cruz, Rio Grande do Norte, Brasil, 2017.

\begin{tabular}{cccc}
\hline \multirow{2}{*}{ Apgar no 10 minuto } & $\mathbf{0 ~ a ~} 6$ & $\mathbf{7}$ a 10 & Não se aplica \\
\cline { 2 - 4 } & $20 \%(\mathrm{n}=31)$ & $76,5 \%(\mathrm{n}=153)$ & $8 \%(\mathrm{n}=16)$ \\
\cline { 2 - 4 } Apgar no $5^{\circ}$ minuto & $\mathbf{0 ~ a ~} 7$ & $\mathbf{8}$ a 10 & Não se aplica \\
\cline { 2 - 4 } & $14 \%(\mathrm{n}=28)$ & $78 \%(\mathrm{n}=156)$ & $8 \%(\mathrm{n}=16)$ \\
\hline \multirow{2}{*}{ Criança com baixo peso ao nascer } & \multicolumn{2}{c}{$\mathbf{1 . 6 1 5}$ a 2.155g } & 2.190 a 2.480g \\
\cline { 2 - 4 } & \multicolumn{2}{c}{$54,5 \%(\mathrm{n}=6)$} & $45,5 \%(\mathrm{n}=5)$ \\
\hline
\end{tabular}

Relacionado aos cuidados ofertados pela equipe de saúde, tratando-se do estímulo ao vínculo materno/infantil, Tabela 3, 90,0\% das mães relataram que permaneceram junto ao seu filho no mesmo quarto após o parto; em relação ao tempo de amamentação ofertada à criança logo após o nascimento, $75,4 \%$ afirmou amamentar seu filho logo após o parto. Ainda 95,0\% afirmou que a criança realizou o teste do pezinho, como preconizado. 


\section{ciência plural}

Tabela 3 - Cuidados ofertados pela equipe de saúde na primeira hora de vida ao último mês em relação a pesquisa. Santa Cruz, Rio Grande do Norte, Brasil, 2017.

\begin{tabular}{cccc}
\hline \multirow{2}{*}{$\begin{array}{c}\text { Mãe e Criança permaneceram no mesmo quarto } \\
\text { no pós-parto }\end{array}$} & Sim & Não & Não se aplica \\
\cline { 2 - 4 } Amamentação nas primeiras horas de vida & $90,0 \%(n=180)$ & $9,0 \%(n=18)$ & $1,0 \%(n=2)$ \\
\cline { 2 - 4 } & Sim & Não & Não se aplica \\
\hline \multirow{2}{*}{ Realizaram o teste do pezinho } & $75,4 \%(n=150)$ & $23,6 \%(n=47)$ & $1,0 \%(n=2)$ \\
\cline { 2 - 4 } & \multicolumn{2}{c}{$\operatorname{Sim}$} \\
\hline
\end{tabular}

Por fim, quando questionadas sobre as condições de saúde no primeiro mês de vida, Tabela 4, a maioria da amostra, 92,5\%, relatou que suas crianças não foram internadas por nenhum tipo de complicação; das que relataram algum tipo de internação, a maioria possuiu idade menor de 7 dias de vida. Sobre o motivo da internação, 2,5\% relataram por motivo a icterícia. No que se referem ao tempo que a criança passou internada, $4,5 \%$ das crianças possuíram entre 1 a 8 dias de permanência; sendo 6,0\%, entretanto, maioria entre as que possuíram internamento, internada em hospitais público/SUS do seu município. Ao serem questionadas se a criança possui plano de saúde, 97,5\% relataram que não.

Tabela 4 - Manutenção da saúde infantil no primeiro mês de vida. Santa Cruz, Rio Grande do Norte, Brasil, 2017.

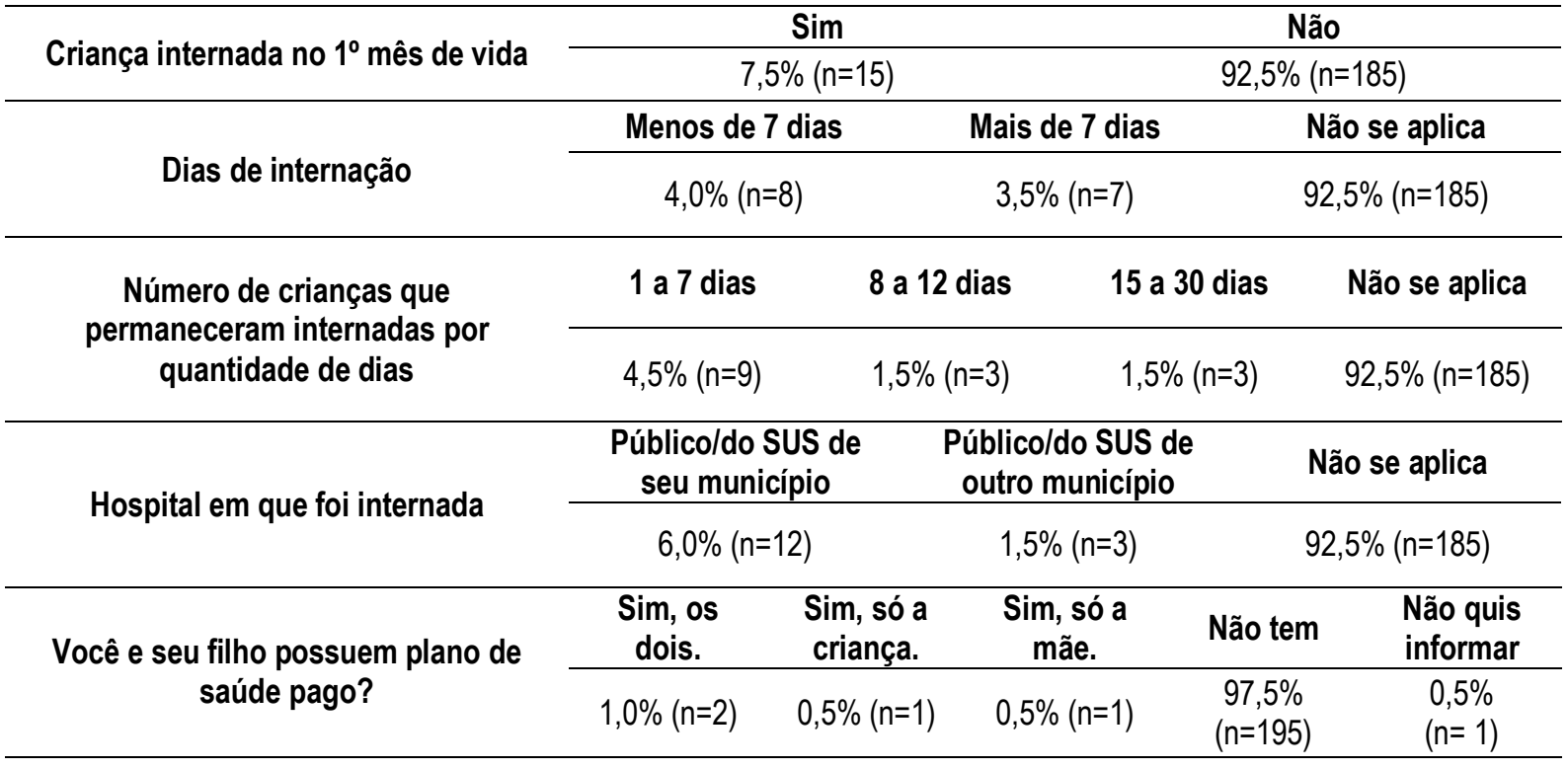




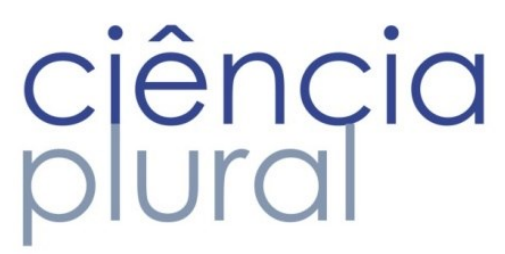

\section{DISCUSSÃO}

Relacionado aos dados de caracterização da amostra, Tabela 1, destaca-se a escolaridade materna, como temática em ampla discussão, uma vez que tem constituído-se como um fator condicionante para 0 crescimento e desenvolvimento infantil, e deve ser um ponto considerado em toda assistência à saúde da criança, de modo que nesta pesquisa identificou-se que a amostra, em maioria, possuía ensino fundamental/médio completo.

Estudo ${ }^{7}$ discute que o nível de escolaridade materna configura-se como um dos fatores que interferem diretamente no crescimento e desenvolvimento psico-morfofuncional da criança entre zero a três anos e ainda, identificou-se que mães com escolaridade inferior a quatro anos eram mais propensas a terem filhos com baixo peso, assim, um nível de escolaridade mais elevado fornece maior facilidade a um ambiente propício para que a criança possa obter um bom desenvolvimento.

Portanto, destarte que os profissionais dos serviços de saúde devem desempenhar um papel essencial frente a essa situação, sendo a educação em saúde uma ferramenta importante para a efetivação do cuidado. Desenvolver métodos práticos que facilitem a compreensão fazendo uso de uma linguagem acessível aos usuários confere um caráter positivo às ações de promoção e prevenção da saúde e consolida uma assistência resolutiva dos serviços.

Frente a isto, ressalta-se que a atenção individualizada pautada por ações que considerem as singularidades que cada uma traz, precisam nortear o exercício dos profissionais, uma vez que entender 0 contexto social em que esses usuários se inserem é de grande importância, além do que uma vigilância continua durante toda essa fase, a fim de detectar possíveis problemas, minimiza os riscos e melhora a qualidade de vida das crianças assistidas.

Em relação à avaliação do índice de Apgar, observado na Tabela 2, esta é realizada no $1^{\circ} \mathrm{e} 5^{\circ}$ minutos após o nascimento, a partir das características apresentadas pelos recém-nascidos, como respiração, tônus muscular, batimentos cardíacos, cor da pele e resposta a estímulos nervosos, permitindo obter sua classificação quanto à presença ou não de anóxia e avaliar os efeitos de eventual reanimação necessária8.

Um estudo realizado ${ }^{9}$ mostrou que crianças que tiveram baixo índice de Apgar no primeiro minuto após o nascimento, possuem grande chance de ir a óbito no período neonatal, pois dos 16 bebes que foram a óbito nos primeiros 28 dias de vida, 15 apresentaram no primeiro minuto, índice de Apgar de 0 a 6 ocasionado por anóxia intraparto grave ou moderada. 


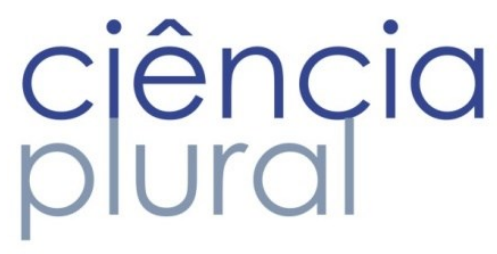

Quanto aos resultados obtidos nesta pesquisa, sobre a porcentagem de nascidos vivos que apresentaram valor de apgar $<7$ e $>7$ no $1^{\circ}$ e no $5^{\circ}$ minuto, observou-se que apgar $<7$ tem apresentado constante diminuição quando comparado $01^{\circ}$ e $05^{\circ}$ minuto. Ressalta-se ainda que a maioria da amostra apresenta valor de apgar $>7$ no $1^{\circ} \mathrm{e}$ apgar $>8 \mathrm{no} 5^{\circ}$ minuto, que revelam-se como fator positivo para a saúde da criança.

Relacionado ao baixo peso ao nascer, nesta pesquisa houve representavidade de 5,5\%. Este representa um dos mais importantes fatores de predição da sobrevida infantil. A OMS ${ }^{10}$ classifica o peso ao nascer em Baixo Peso (menos de 2.500g), Muito Baixo Peso (menos de 1.500g) e Extremo Baixo Peso (menos de $1.000 \mathrm{~g}$ ) ao nascer. Logo, quanto menor for o peso ao nascer, maior será o risco para a mortalidade infantil, ocorrendo principalmente no período neonatal.

Em estudo realizado por Sarinho et al. ${ }^{11} \mathrm{o}$ baixo peso ao nascer foi apontado como o principal fator relacionado à morte neonatal estando ele evidenciado pela prematuridade ou não. 0 risco de morte apresentado pelos recém-nascidos de baixo peso foi 30 vezes maior se comparados com os nascidos com peso maior que $2.500 \mathrm{~g}$.

Outro ponto importante trata-se do reconhecimento e o contato pele a pele precoce mãe-filho, Tabela 3 , como um momento único e especial no processo de nascimento garantido pelos profissionais de saúde na assistência. Este contato imediato demonstra a modificação assistencial que vem ocorrendo nos serviços de saúde nos últimos anos, fundamentado na humanização como base de tal prática, porém, ainda há situações desfavoráveis, pois, depende da sensibilidade profissional frente aos valores subjetivos que estão presentes em meio aos procedimentos e a conclusão do parto essa prática não é adotada ${ }^{12}$.

Observou-se que o contato entre mãe e filho ocorrido logo após o parto foi relatada pela maioria da amostra avaliada, demonstrando que essa prática tem sido rotina no município estudado, entretanto destaca-se a necessidade de avaliar fatores que tem dificultado tal prática na totalidade de usuárias do serviço. Estudo ${ }^{13}$ a partir de levantamento bibliográfico ratifica que mãe e filho estavam aptos e sensíveis, logo após o parto, para a ocorrência dessa interação, possibilitando fortificar o elo existente, no qual influenciaria os envolvidos em sua vivência e o seu contato interpessoal estabelecido ao longo de suas vidas.

Outro aspecto bastante importante que se destaca como beneficio do contato imediato influenciando de forma positiva ao vínculo mãe-filho é a amamentação nas primeiras horas de vida do recém-nascido, como observou-se em maior parcela desta amostra. Ressalta-se que além dos aspectos nutricionais e imunológicos integrados à amamentação ${ }^{14}$, tal prática promove e apoia 0 aleitamento materno exclusivo reforçando cada vez mais as políticas e ações pautadas a propiciar um início de vida saudável. 


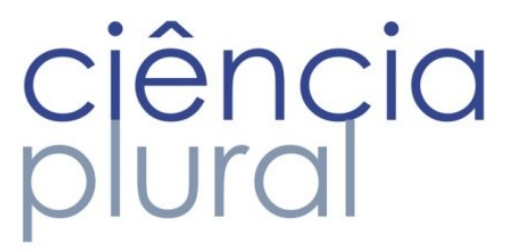

Com isso torna-se essencial adotar práticas que abordem aspectos essenciais do aleitamento materno logo após o nascimento, capacitando toda a equipe de saúde a estrem aptos em esclarecer todas as dúvidas que possam surgir no momento inicial da amamentação, conscientizando a mãe sobre os benefícios excepcionais que 0 aleitamento materno proporciona e assim contribuir de forma ativa com 0 fortalecimento do vínculo mãe/filho construindo um ambiente favorável para o bom desenvolvimento da sua criança.

Relacionado a realização do teste do pezinho, Oliveira e Souza ${ }^{15}$, destaca que por vezes há uma falha na orientação durante o pré-natal por parte dos profissionais de saúde quando não instruem a gestante quanto a sua importância, obtendo como consequência, que estas mulheres não compreendem a real importância em se realizar o teste do pezinho nos recém-nascidos, que configura-se como importante procedimento, através do qual torna possível identificar, de forma precoce, doenças como hipotireoidismo congênito, hiperfenilalaninemias, hemoglobinopatias, e fibrose cística ${ }^{16}$.

Contando que tal prática configura-se como cuidado essencial e que deve ser estimulada pelos serviços de saúde, no município em questão, a maioria da amostra o realizou, destacando que embora não tenha sido em sua totalidade, evidencia-se que ainda sim, esta prática tem sido considerada relevante para o cuidado a saúde infantil, refletindo a qualidade da assistência prestada.

A atenção à saúde da criança representa um campo prioritário dentro dos cuidados à saúde das populações. Para que essa se desenvolva de forma mais efetiva e eficiente, além do conhecimento sobre as características relacionadas à morbi-mortalidade, tais como aspectos biológicos, demográficos e socioeconômicos, é importante salientar o papel que desempenham os serviços e o sistema de saúde ${ }^{6}$.

Desse modo, destaca-se os dados relacionados a manutenção da saúde infantil no primeiro mês de vida, Tabela 4, onde observou-se que bons indicadores de saúde da população infantil, uma vez que a maioria da amostra não relata internação hospitalar no primeiro mês de vida, e quando houve, possuiu duração inferior a 7 dias, em serviços de saúde do Sistema Único de Saúde do seu município.

Ressalta-se que os serviços de puericultura na APS destacam-se quanto ao constante monitoramento do crescimento e desenvolvimento infantil, com foco no diagnóstico precoce de patologias, na promoção a saúde e prevenção de doenças ${ }^{17}$. De modo, que compete atuar como porta de entrada do paciente no sistema de atenção à saúde, disponibilizando uma assistência integral ao usuário, voltada a solucionar as demandas mais frequentes em sua região de abrangência, otimizando a utilização dos demais níveis de atenção.

Sugere-se ainda, que as melhorias da promoção à saúde da criança estão ligadas ao serviço de APS, por seu caráter individual, familiar e coletivo bem como por possibilitar os recursos necessários para 
os profissionais, tanto quanto a qualificação destes para o gera bom desenvolvimento das atividades e resolutividade das necessidades do usuário.

Os cuidados prestados à criança em seus primeiros momentos de vida constituem um importante aspecto que, quando desempenhado adequadamente, tende a fortalecer o elo entre a família/criança e os serviços de saúde. O programa de puericultura propõe o acompanhamento da saúde infantil, a fim de garantir um crescimento saudável sendo responsável pela promoção, prevenção e recuperação dos indivíduos nessa fase da vida, além do mais, tudo isso associado ao acolhimento adequado e comprometimento profissional aumenta a perspectiva de se obter uma concreta adesão das práticas em saúde.

\section{CONCLUSÕES}

Observou-se que as práticas assistenciais foram realizadas em sua maioria, destacando o comprometimento dos profissionais atuantes nos serviços de saúde, o que confere aos serviços uma boa qualidade frente às demandas solicitadas. Sob uma ótica multidimensional esta qualidade mostrou grandes avanços, porém as fragilidades e desafios ainda existentes, demonstram a necessidade de estratégias para implementação de medidas estratégicas em busca de melhores resultados.

Cabe destacar como limitações deste estudo, o viés de memória de mães e responsáveis. Entretanto, este pode ser diminuído uma vez que se trata de evento importante na vida materna, o nascimento do filho e os eventos que nele ocorrem.

Ressalta-se ainda a importância da compreensão dos usuários sobre as orientações repassadas quanto à realização dos exames de triagem, o comparecimento às consultas, a realização dos cuidados prescritos, dentre outros, o que os torna corresponsáveis pela eficácia dos cuidados direcionados à criança, uma vez que após a alta hospitalar, emerge o cuidado no ambiente familiar, possuindo a rede da APS, pelo vinculo com o usuário e as atividades propostas, como orientadora do cuidado.

O presente estudo torna-se relevante, à medida que pode contribuir para estimular a elaboração de novas estratégias de melhoria no cuidado à saúde da criança, buscando fortalecer as iniciativas de promoção e recuperação da saúde e prevenção de agravos. Através do monitoramento de ações e resultados pode-se conhecer as reais necessidades do serviço, a qualidade da assistência prestada, fundamentais para adequação de políticas públicas de saúde e implementação de ações, estimulando ainda 0 alcance de indicadores cada vez melhores. 


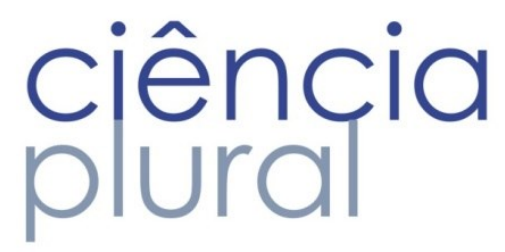

\section{REFERÊNCIAS}

1. Ministério da Saúde (BRASIL). Secretaria de Atenção à Saúde. Departamento de Ações Programáticas Estratégicas. Política Nacional de Atenção Integral à Saúde da Criança: orientações para implementação. Ministério da Saúde. Secretaria de Atenção à Saúde. Departamento de Ações Programáticas Estratégicas. - Brasília : Ministério da Saúde, 2018.

2. Moreira MEL, Gama SGN, Pereira APE, Silva AAM, Lansky S, Pinheiro RS et al . Práticas de atenção hospitalar ao recém-nascido saudável no Brasil. Cad. Saúde Pública [Internet]. 2014; 30( Suppl 1): S128-S139.

3. Smith ER, Hurt L, Chowdhury R, Sinha B, Fawzi W, Edmond KM et al. Delayed breastfeeding initiation and infant survival: A systematic review and meta-analysis. PLoS One. 2017;12(7):e0180722.

4. Matos TA, Souza MS, Santos EKA, Velho MB, Seibert ERC, Martins NM. Contato precoce pele a pele entre mãe e filho: significado para mães e contribuições para a enfermagem. Rev. bras. enferm. [Internet]. 2010 Dec [; 63(6):998-1004.

5. Campos RMC, Ribeiro CA, Silva CV, Saparoll EL. Consulta de enfermagem em puericultura: a vivência do enfermeiro na Estratégia de Saúde da Família. Rev. Esc. Enferm. USP. Out 2011;45(3):566-74.

6. Machado MMT et al. Características dos atendimentos e satisfação das mães com a assistência prestada na atenção básica a menores de 5 anos em Fortaleza, Ceará. Ciênc. saúde coletiva [online]. 2012; 17(11): 3125-33.

7. Silva JLGV, Soares EA, Caetano EA, de Souza Loyola YC, Garcia JAD, Mesquita G. O Impacto da Escolaridade Materna e a Renda Per Capita no Desenvolvimento de Crianças de Zero a Três Anos. Revista Ciências em Saúde. 2011; 1(2): 62-67.

8. Ziegel EE, Cranley MS. Enfermagem Obstétrica. 8.ed. Rio de Janeiro: Guanabara koogan, 1985.

9. Poles K, Parada CMGL. Mortalidade infantil em município do interior do Estado de São Paulo. Rev. Esc. Enferm. USP, São Paulo,2002; 36(1):10-7.

10. Organização Mundial de Saúde (OMS). Classificação Estatística Internacional de Doenças e Problemas Relacionados à Saúde. Décima Revisão. CID-10. Volume 2. Manual de Instruções Tradução Centro Colaborador da OMS para a Classificação de Doenças em Português. 7 ed. São Paulo: Editora da Universidade de São Paulo, 2004.

11. Sarinho SW et al. Fatores de risco para óbitos neonatais no Recife: um estudo caso-controle. Jornal de Pediatria, 2001; 77(4): 294-98. 
12. Santos RAA, Melo MCP, Cruz DD. Trajetória de humanização do parto no Brasil a partir de uma revisão integrativa de literatura. Cadernos de Cultura e Ciência. Mar 2011. 13(2):76-89.

13. Silva PA, Esteves ML, Castro FV. Vinculação mãe bebê. Infad Revista de Psicología. 2013; 2(1):72936.

14. Penna FJ, Nicoli JR. Influência do colostro na colonização bacteriana normal do trato digestivo do recémnascido. Jornal de Pediatria. 2001; v. 77.

15. Oliveira EF, Souza AP. A importância da realização precoce do teste do pezinho: o papel do enfermeiro na orientação da triagem neonatal. Revista Multidisciplinar e de Psicologia. 2017; 11(35): 361-378.

16. Costa SN, Ney BS, Ricardo DC, Eduardo GO, Fábio DC. Triagem neonatal para fenilcetonúria, hipotireoidismo congênito e hemoglobinopatias no recôncavo baiano: avaliação da cobertura em cruz das almas e Valença, Bahia, Brasil. Rev. baiana saúde pública. jul.-set. 2012. 36(3):831-43.

17. Barboza CL, Barreto MS, Marcon SS. Records of childcare in primary care: descriptive study. Online braz j nurs [periodic online]. 2012; 11(2):359-75.

Submissão: $10 / 8 / 2018$

Aceitação: 20/3/2019 